\title{
PERFIL BIBLIOMÉTRICO DA PRODUÇÃO CIENTÍFICA DA REVISTA BRASILEIRA DE APRENDIZAGEM ABERTA E A DISTÂNCIA NO PERÍODO DE 2002 A 2012
}

\author{
Carlos Alberto Serra Negra \\ Centro Universitário do Leste de Minas Gerais \\ casene@terra.com.br \\ Ana Paula Costa e Silva \\ Universidade Católica de Brasília \\ asilva@ucb.br
}

\section{RESUMO}

A modalidade de Educação a Distância tem apresentado um crescimento vertiginoso nos últimos anos e, como consequência, tem apresentado um número de pesquisas e publicações elevado. Além da necessidade de pesquisa e publicação sobre os diversos aspectos que norteiam o tema, é igualmente importante levantar o que se está publicando sobre o assunto. O objetivo da pesquisa foi o de descrever o Perfil Bibliométrico da Produção Científica da Revista Brasileira de Aprendizagem Aberta e a Distância no período de 2002 a 2012. A pesquisa se classifica como descritiva quanto aos objetivos, como bibliográfica para levantamento de referencial teórico e de bibliometria quanto ao procedimento. Os resultados foram apurados após tabulação estatística de onze variáveis de análise. Os resultados permitiram traçar o Perfil Bibliométrico da revista em estudo com um alto nível de profundidade.

Palavras-Chave: Educação a Distância. Bibliometria. Revista Brasileira de Aprendizagem Aberta e a Distância.

\section{BIBLIOMETRIC PROFILE OF THE SCIENTIFIC PRODUCTION OF THE BRAZILIAN REVIEW OF OPEN AND DISTANCE LEARNING IN THE PERIOD 2002 TO 2012}

\begin{abstract}
The distance education modality has presented a dizzying growth in recent years and, as a consequence, has presented a high number of research and publications. In addition to the need for research and publication on the various aspects that guide the theme, it is equally important to rise what is publishing on the subject. The objective of this research was to describe the Bibliometric Profile of the Scientific Production of the Brazilian Review of Open and Distance Learning in the period from 2002 to 2012. The research ranks as descriptive as to the objectives, as for bibliographical survey of theoretical and of Bibliometrics with regard to the procedure. The results were calculated after eleven statistic tabulation analysis variables. The results allowed Bibliometric profile of revised study with a high level of depth.
\end{abstract}

Keywords : Distance education. Bibliometrics. Brazilian Review of Open and Distance Learning. 


\section{INTRODUÇÃO}

O Ensino a Distância é um fenômeno contemporâneo que teve início, segundo alguns autores, a partir dos anos de 1800 e grande crescimento mundial a partir do término da segunda guerra mundial (1946). Sua transição de ensino para Educação a Distância (EAD) já é um fenômeno da pós-modernidade nos termos previstos por Gidens (1991; 2002) e com o uso da Tecnologia da Informação e Comunicação (TIC) nos preceitos da sociedade em rede de Castells (1999).

Ainda que a exploração histórica do desenvolvimento da educação a distância tenha discrepância entre autores, o sistema de gerações proposto por Moore e Kearsley (2007) é didaticamente recomendável, considerando: Primeira Geração - estudo por correspondência; Segunda Geração - transmissão por rádio e televisão; Terceira Geração - abordagem sistêmica da Universidade Aberta; Quarta Geração teleconferência; Quinta Geração - aulas virtuais baseadas na internet.

Os autores Perrotti e Vigneron (2003), citados por Carpes et al. (2011, p. 2) são de opinião que o ensino a distância possui especificidades próprias e não pode ser visto como uma simples cópia do modelo presencial tradicional, sendo necessários alguns cuidados, a fim de atender adequadamente à nova demanda social que surge a partir deste modelo. Assim, a EAD requer a formação de um novo educador, de novas abordagens pedagógicas, de novas tecnologias educacionais e, por não dizer, de um novo aluno.

A expansão e crescimento da EAD no Brasil foram acompanhados também da expansão das pesquisas sobre essa temática. Diferentes áreas do conhecimento, em uma visão multi e interdisciplinar, têm sido atraídas por essa modalidade de ensino que proporciona inúmeras vantagens, tais como flexibilidade de estudo, material próprio de ensino, tutores e professores capacitados e aprendizagem intermediada por internet.

Por conseguinte, o problema de pesquisa é dado pela seguinte questão: como se configura o Perfil Bibliométrico da Produção Científica da Revista Brasileira de Aprendizagem Aberta e a Distância (RBAAD) no período de 2002 a 2012?

A pesquisa justifica sua importância por, principalmente, três motivos: primeiro porque o tema „educação a distância ${ }^{\text {ee }}$ ainda precisa ser muito explorado em termos de experiências concretas, pesquisas e publicações; segundo, pela necessidade de levantamentos bibliométricos envolvendo todos os aspectos da EAD ou de periódicos específicos; terceiro porque a pesquisa pode contribuir para aperfeiçoamento da Revista Brasileira de Aprendizagem Aberta e a Distância (RBAAD).

A escolha de RBAAD, em detrimento a outras publicações que veiculam a temática de EAD, deu-se pelos seguintes motivos: muitos anos de publicação ininterrupta, abrangência internacional, característica interdisciplinar, disponibilização de todos os seus artigos na internet, além do fato de a RBAAD vir apresentando um processo evolutivo de normas, conteúdos e layout.

O objetivo da pesquisa consiste em descrever o Perfil Bibliométrico da Produção Científica da Revista Brasileira de Aprendizagem Aberta e a Distância (RBAAD) no período de 2002 a 2012. 


\section{REVISÃO DE LITERATURA}

\subsection{Elementos Técnicos e Metodológicos de Estudos Bibliométricos}

Do ponto de vista histórico, a bibliometria é uma técnica recente nos estudos sobre produção científica, porque levantar, analisar, acompanhar, mapear e avaliar as publicações científicas com o uso de procedimentos estatísticos próprios, especialmente quanto às tendências de área temáticas, aspectos técnicos e procedimentos metodológicos, requer uma visão diferenciada dos trabalhos publicados a partir de pesquisas realizadas.

Consistindo na aplicação de técnicas estatísticas e matemáticas para descrever aspectos da literatura e de outros meios de comunicação (análise quantitativa da informação), a bibliometria foi originalmente conhecida como bibliografia estatística (termo cunhado por Hulme em 1923), sendo o termo 'Bibliometria' criado por Otlet em 1934 no seu Traité de Documentation (ARAÚJO, 2006). Contudo, o termo apenas se popularizou em 1969, a partir de um artigo de Pritchard que discutia a polêmica "bibliografia estatística ou bibliometria?" (VANTI, 2002, p. 153, apud ARAÚJO, 2006). A diferença essencial entre a tradicional bibliografia e a bibliometria é que esta utiliza mais métodos quantitativos do que discursivos. Assim, a utilização de métodos quantitativos na busca por uma avaliação objetiva da produção científica é o ponto central da bibliometria.

Kobashi e Santos (2008), citados por Moura, Dallabona e Lavarda (2012, p. 97-125), apresentam que a bibliometria é uma metodologia de avaliação de trabalhos científicos que apresentam as mesmas particularidades. Por meio dela, pode-se, por exemplo, identificar a quantidade de trabalhos sobre um determinado assunto; as publicações em uma data precisa; publicações por um autor ou por uma instituição ou difundidos por um periódico científico. A verificação e análise dos indicadores bibliométricos permite quantificar a produção técnica e científica. Segundo Saes (2000), citado por Moura, Dallabona e Lavarda (2012, p. 97-125), os indicadores bibliométricos são empregados para analisar o tamanho, crescimento e distribuição da bibliografia científica (livros, revistas, patentes e outros), e analisar os processos de geração, propagação e uso da literatura científica.

O desenvolvimento da bibliometria está centrado em três leis principais: a lei de produtividade científica de autores de Lotka; a lei de dispersão de periódicos de Bradford e a lei de frequência de palavras de Zipf. (ARAÚJO, 2006).

\subsection{Estudos Bibliométricos em Educação a Distância no Brasil}

Para levantamento de estudos bibliométricos sobre educação a distância, foram pesquisados 4 (quatro) base de dados: Scirus for scientific Information Only; Science Direct, Scielo - Scientific Electronic Library Online e o Google Acadêmico.Os termos de busca utilizados foram os seguintes: „Análise Bibliométrica de Educação a Distância $^{e e}$, „Estudo Bibliométrico de Educação a Distância ${ }^{\text {ee }}$,Perfil Bibliométrico de Educação a Distância" e „Bibliométrico + Educação a Distância"e e teve como parâmetro de busca os 100 (cem) primeiros resultados (10 páginas indicando dez sites por página) nos últimos 5 (cinco) anos. Os principais trabalhos são os a seguir mencionados no quadro 1. 
Quadro 1 - Estudos Bibliométricos de Educação a Distância dos últimos 5 anos

\begin{tabular}{|c|c|c|c|}
\hline $\begin{array}{c}\text { Ano do } \\
\text { Artigo }\end{array}$ & Autores & Período Pesquisa & Amostra \\
\hline 2009 & $\begin{array}{c}\text { Barbara C. Neves } \\
\text { José Carlos S. dos Santos } \\
\text { Neubler Nilo R. da Cunha }\end{array}$ & $2002-2006$ & 10 periódicos \\
\hline 2010 & $\begin{array}{c}\text { Fernando José Spanhol } \\
\text { Kelly Cristina B. T. Tosta } \\
\text { Kamil Giglio } \\
\text { Patrícia de Sá Freire }\end{array}$ & $1998-2008$ & 189 artigos \\
\hline 2011 & $\begin{array}{c}\text { Cleyton V. C. Magalhães } \\
\text { Ronnie E. S. Santos } \\
\text { Jorge S. Correia } \\
\text { Ellen P. R. Souza }\end{array}$ & $2001-2009$ & 112 artigos \\
\hline 2011 & $\begin{array}{c}\text { Aletéia de Moura Carpes } \\
\text { Thiago Antônio Beuron } \\
\text { Thiago Reis Xavier Ana } \\
\text { Paula Perlin } \\
\text { Vitor Francisco Schuch Júnior }\end{array}$ & $1998-2010$ & 44 artigos \\
\hline 2012 & $\begin{array}{l}\text { Fernanda Roda Cassundé } \\
\text { Nildo Cassundé Júnior }\end{array}$ & $19980-2011$ & 48 artigos \\
\hline 2012 & $\begin{array}{l}\text { Suelen Haidar Ronchi } \\
\text { Sandra Rolim Ensslin } \\
\text { Leonardo Ensslin } \\
\text { Sergio Murilo Petri }\end{array}$ & $2004-2009$ & 18 artigos \\
\hline 2013 & $\begin{array}{l}\text { Henrique S. B. Cavalcanti Júnior } \\
\text { Isabela Neves Ferraz }\end{array}$ & 2002-2011 & 22 artigos \\
\hline 2013 & $\begin{array}{c}\text { Stella Cecilia Duarte Segenreich } \\
\text { Luiz Felipe Camêlo de Freitas }\end{array}$ & $1978-2009$ & $\begin{array}{l}247 \text { trabalhos } \\
\text { monográficos }\end{array}$ \\
\hline
\end{tabular}

Fonte: Autores

Pela análise do quadro 1, é possível afirmar que os estudos bibliométricos sobre EAD no Brasil têm sido elaborados dentro da perspectiva da construção coletiva do conhecimento, com mais de dois autores; e que as amostras das pesquisas estão adequadas aos estudos propostos, mas havendo ainda produção bilbiométrica sobre a temática EAD nos últimos cinco anos.

\section{MATERIAIS E MÉTODOS}

Quanto ao seu objetivo, a pesquisa se caracteriza como descritiva. Segundo Gil (1996, p. 46), as pesquisas descritivas têm como objetivo primordial a descrição das características de determinada população ou fenômeno ou, então, o estabelecimento de relações entre variáveis.

Quanto à abordagem, a pesquisa é classificada como quantitativa. De acordo com Diehl e Tatim (2004, p. 72) ela se caracteriza pelo uso da quantificação tanto na coleta quanto no tratamento das informações por meio de técnicas estatísticas, desde as mais simples, como percentual, média, quantidade, moda, desvio-padrão, até às mais complexas como coeficiente de correlação, análise de regressão, etc. 
No que diz respeito ao estudo empírico, a pesquisa adotou o procedimento de análise bibliométrica dos artigos selecionados. Nicholas (1978) citado por Avelar, Santos e Mesquita (2011) conceitua análises bibliométricas como uma descrição estatística ou quantitativa da literatura de uma determinada área. Este tipo de análise pode ser utilizado para diversos fins tais como: identificar as tendências e o crescimento do conhecimento de uma área; identificar as revistas do núcleo de uma disciplina; prever as tendências de publicação; estudar a dispersão e obsolescência da literatura científica; e medir o crescimento de determinadas áreas e o surgimento de novos temas.

O objeto e população da pesquisa são os materiais que compõem a Revista Brasileira de Aprendizagem Aberta e a Distância (RBAAD) publicada on line pela Associação Brasileira de Educação a Distância (ABED) no site http://www.abed.org.br/revistacientifica/_brazilian/default.htm. Dessa forma, de um universo (população) de 131 publicações em 11 (onze) anos de revista, sendo: 108 sob a forma de artigos, 11 sob a forma de resenhas e 12 sob a forma de materiais técnicos. Tendo em vista que para materiais escritos de outras formas, que não seja artigo técnico ou científico, não existem normalização, optou-se como amostragem somente os materiais publicados na forma de artigo. Estes têm sua estrutura normalizada pela Associação Brasileira de Normas Técnicas (ABNT). Conforme NBR-6022 de maio de 2003.

Com base no estudo e na adaptação das classificações de Cavalcanti Júnior e Ferraz (2013), foram consideradas as variáveis de análise relacionadas no quadro 1.

Quadro 1 - Variáveis de Análise Bilbiométrica

\begin{tabular}{|l|l|}
\hline 1. Quantidade de Páginas & 7. Técnica de Coleta de Dados \\
\hline 2. Quantidade de Autores & 8. Metodologia - Natureza da Pesquisa \\
\hline 3. Natureza do Estudo & 9. Procedimento da Pesquisa \\
\hline 4. Metodologia - Abordagem & 10. Temática ou Assunto do Artigo \\
\hline 5. Metodologia - Norma de Citações & 11. Elaboração do Resumo \\
\hline 6. Referências - Norma aplicada & \\
\hline
\end{tabular}

Fonte: Autores

Os dados dos 108 artigos da amostra foram, concomitantemente a leitura e análise, tabulados em planilha eletrônica (Excel 2010 a Microsoft) e apresentados sob a forma de tabelas para melhor visualização dos dados, mostrando, para cada variável de análise a frequência absoluta e relativa.

\section{APRESENTAÇÃO E ANÁLISE DE RESULTADOS}

\subsection{Revista Brasileira de Aprendizagem Aberta e a Distância (RBAAD)}

A Revista Brasileira de Aprendizagem Aberta e a Distância (RBAAD) é uma publicação periódica on line com foco em pesquisa, desenvolvimento e prática da educação a distância em todos os níveis educacionais, formais e informais, e em todas as tecnologias disponíveis.

Com uma esfera de ação internacional, a RBAAD publica artigos voltados para os êxitos e desafios em educação a distância para pessoas de todas as idades, grupos ou níveis, nos sistemas formal e informal de educação. A revista aceita e publica artigos em 
três idiomas - português, inglês e espanhol, sendo que a política e a seleção de artigos são determinadas pelos objetivos editoriais da $\mathrm{ABED}$, pelo grupo editorial da revista $\mathrm{e}$ por orientações específicas (RBAAD, 2103).

A RBAAD aceita textos sobre educação a distância de pesquisadores e praticantes de todas as partes do mundo, mas sempre que esses projetos de trabalho sejam de especial relevância para os respectivos países. Os artigos podem ser filosóficos e/ou de análises qualitativas e quantitativas; podem ter o formato de estudo de casos, pesquisas ou relatórios de progresso de projetos em andamento. Resenhas de livros, relatos de congressos, URLs para homepages de inovações na educação a distância, anúncios de conferências e publicações de cartas para o editor também são bem-vindas (RBAAD, 2103).

A RBAAD é uma publicação indexada com o ISSN de número 1806-1362 e uma classificação QUALIS B3 como periódico interdisciplinar e B4 como periódico de educação (CAPES, 2013).

\subsection{Resultados da Pesquisa}

A análise da quantidade de páginas por artigo tende a evidenciar além de um padrão para periódicos (revistas e eventos científicos), a necessidade de uma síntese na escrita e no nível de aprofundamento dado às questões de pesquisa. Os resultados apurados mostram que $61 \%$ dos artigos têm entre 8 a 14 páginas, $16 \%$ entre 3 a $7,14 \%$ entre 15 a 21 e $10 \%$ acima de 22 páginas.

$\mathrm{O}$ número de autores que publicam cada artigo evidencia redes e parcerias entre autores. $\mathrm{Na}$ medida em que mais autores publicam em conjunto, percebe-se que a área é investigada por grupos de pesquisa ao invés de autores individuais. A colaboração entre autores vem sendo vista internacionalmente como um dos indicadores de qualidade da pesquisa, principalmente em temas interdisciplinares (SUBRAMANYAM, 1983 apud RIBEIRO et al. 2012, p. 47).

No que se refere a quantidade de autores por artigo, apurou-se que a predominância é de autoria individual (54\%) seguida por dois autores por artigo (24\%), três autores $(12 \%)$ mais de quatro com $7 \%$ e $1 \%$ sem autoria, demonstrando tratar de uma área ainda pouco explorada em termos de construção coletiva do conhecimento e por tratar mais de opiniões e pesquisas individuais que institucionais.

No que se refere ao tipo de estudo, há discrepâncias entre pesquisas realizadas com a temática „Educação a Distância“e. Os dados apurados pela pesquisa apontam que $60 \%$ foram estudos teóricos e $40 \%$ estudos empíricos. Na pesquisa de Cavalcanti Junior e Ferraz (2013), os estudos empíricos obtiveram $72 \%$ e os bibliográficos $28 \%$. Resultado distinto é apontado por Carpes et al. (2011), nos anais dos eventos da ANPAD, já que eles encontraram somente artigos empíricos sobreEAD.

Segundo Gil (1996), as pesquisa quanto aos objetivos são classificadas como Exploratórias, Descritivas e Explicativas. Os dados levantados e inseridos na tabela 5 evidenciam que $95 \%$ dos artigos analisados não tiveram mencionados a classificação da respectiva pesquisa. Isto mostra a pouca importância dada aos autores para classificações mais pormenorizadas da pesquisa. Com a redução da quantidade de 
páginas por artigo, a metodologia da pesquisa acabou sendo preterida a outras partes do artigo. A maioria dos autores escreve pouco na metodologia da pesquisa de modo que muita das vezes nem é possível replicar a pesquisa por falta de detalhamento procedimental. $\mathrm{Na}$ pesquisa forma determinados que 95\% (103 artigos) não mencionaram a classificação, 3\% fora Exploratórias e 2\% Descritivas. Na pesquisa de Carpes et al. (2011), 40,91\% dos estudos foram de abordagem descritiva, 38,64\% exploratórias-descritiva, $18,18 \%$ exploratória e $2,27 \%$ causal.

As citações em textos científicos no Brasil adota a norma NBR - 10520 de agosto de 2002. Pode-se adotar o sistema numérico ou o sistema autor-data para identificação do citado. O principal elemento de reconhecimento de que uma citação está de acordo com a norma brasileira é chamada de „regra do autor ${ }^{\text {"e }}$, onde o sobrenome do autor fora do parêntese, que indica ano e página, é minúsculo e quando dentro do parêntese é todo maiúsculo, forma bem diferente de outras normas internacionais de citações. Exemplos: Silva (2012, p. 34) e (SILVA, 2012, p. 34). Os resultados apurados estão demonstrados Da seguinte forma: do total dos artigos da amostra, cerca de 56\% trabalharam com outras normas, indicadas por dois pontos separando ano de página (:) e não a forma brasileira da palavra página abreviada em minúsculo com um ponto (p.) e também pelo uso de sobrenome todo minúsculo dentro ou fora do parêntese. $41 \%$ dos artigos apresentaram conformidade com a norma brasileira, sendo 35 artigos no sistema autordata e 3 artigos no sistema numérico.

Um ponto a ser discutido são artigos que não apresentaram nenhuma citação, um total de 10 artigos. De certa forma, a citação é um elemento que corrobora e prova as afirmações do autor do artigo. Não ter nenhuma citação no artigo pode ser uma demonstração de profundo conhecimento acerca do assunto, ou de desleixo, ou de plágio velado.

A análise das referências bibliográficas, quer em seus aspectos quantitativos (quantos?), quer no qualitativo (quem?), é um fator importante para o desenvolvimento e a continuidade da ciência, "pois impacta no reconhecimento de pesquisadores por seus pares; estabelece direitos de propriedade e de prioridade da publicação; e constitui importantes fontes de informação, mostrando com isso a literatura que é indispensável para o trabalho dos pesquisadores" (FORESTI, 1990 apud RIBEIRO, 2012, p. 51).

A norma brasileira que regulamenta a formatação de referências é a NBR 6023, de agosto de 2002. Deve haver relação direta entre citação e referências, de forma que para cada autor citado deve haver uma ou mais referências desse autor. De forma análoga, toda referência bibliográfica tem que estar citada no texto. Os resultados encontrados nos levantamento de dados mostra que a maioria dos artigos publicados na revista (62\%) trabalhou com normas internacionais de referências, não adotando o padrão brasileiro (ABNT); 35\% adotaram a norma da ABNT e 3\% dos textos não tiveram nenhuma referencia bibliográfica. Isto pode ser explicado pelos seguintes fatores: a) Não exigência nas normas de publicação da revista de utilização da ABNT; b) Há muitos autores estrangeiros publicando na revista, já que ela é de caráter internacional; c) como a RBAAD não exige ineditismo de publicação, o mesmo artigo pode ter sido apresentado a outro periódico e dessa forma, ter utilizado padrões mais consagrados em outros sistemas para atender determinada área de conhecimento ou outra norma de publicação. 
Pelas informações redacionais de muitas referências em vários artigos pode-se conhecer qual norma o autor trabalhou, mas muitas por misturarem estilos não permitiram seu reconhecimento. As normas identificadas nos artigos têm como modelos as normas citadas e cujos exemplos foram retirados de Serra Negra (2011): Norma da APA Associação de Psicologia Americana, Norma de Chicago, Norma de Vancouver e Norma da MLA - Morden Language Association.

Quanto às técnicas de levantamento de dados, cerca de $87 \%$ dos artigos da amostra não relataram como os dados foram apurados, $4 \%$ utilizaram entrevistas, $5 \%$ observação e $5 \%$ questionário. É importante que as pesquisas, ao serem publicadas, evidenciem a forma de levantamento de dados, inclusive com detalhamento dos procedimentos de pesquisa para que as mesmas possam. No futuro, serem passíveis de reprodução e análise comparativa.

No que se refere à natureza das pesquisas, estudo de Carpes et al. (2011) mostram uma frequência de $45,45 \%$ quantitativa, $31,82 \%$ qualitativa e $22.73 \%$ quali-quantitativa para a produção científica brasileira de educação a distância. Já na pesquisa de Cavalcanti Junior e Ferraz (2013), os artigos analisados mostraram que 56\% dos estudos são qualitativos, $25 \%$ quantitativo e $19 \%$ mistos. Os resultados da RBAAD apurados mostram que $94 \%$ dos artigos ali publicados não declararam a natureza das suas pesquisas, e $6 \%$ estão entre pesquisas de natureza qualitativa, quantitativa e qualiquantitativa. Pode-se até chegar a conclusão da natureza pela leitura dos textos dos artigos, mas o que foi apurado era se o texto trazia expressamente a natureza dos artigos.

Outro fator importante de análise foi com alusão ao procedimento adotado na pesquisa. Os dados apurados expressam que $62 \%$ das publicações da revista são procedimentos bibliográficos, seguidos de $21 \%$ de experimental e $16 \%$ estudos de casos e $1 \%$ de pesquisa documental. Na pesquisa de Cavalcanti Junior e Ferraz (2013) evidencia que a estratégias de investigação foram $56 \%$ de estudo de caso, $15 \%$ de levantamento e em 19\% não foi explicitado o procedimento adotado. Na pesquisa de Carpes et al., (2011) pesquisas survey foram $52,27 \%$, estudo de caso $31,82 \%$ e experimental em $2,27 \%$ e outras formas em 13,64\%.

Elemento obrigatório e importante em um artigo científico é o seu resumo. Este é normalizado pela norma NBR 6028 de 2003 da ABNT - Associação Brasileira de Normas Técnicas. Esta norma prevê a seguinte estrutura para os resumos de publicações científicas: contextualização do tema, objetivo geral, metodologia da pesquisa, resultados e conclusão. Nos levantamentos dos dados da pesquisa apurou-se que 1 artigo não teve resumo e dos que tiveram, cerca de $86 \%$ tinham resumo de forma incompleto e que $13 \%$ tiveram resumos completos de acordo com a norma da ABNT.

Como a RBAAD é um periódico interdisciplinar, foram apuradas as temáticas recorrentes nos artigos pela análise do título ou da dedução dos próprios textos. As escolhas das áreas temáticas foram adaptadas da pesquisa de Spanhol et al. (2013) acerca das áreas afins abordadas nos estudos por eles analisados. As áreas temáticas mais recorrentes na revista apuradas foram: 22\% de Propostas Pedagógicas, 18\% de Tecnologia da Informação e Comunicação (TIC), 10\% descrição de EAD em outros países, 9\% de Design Instrucional e $8 \%$ tratando de assuntos envolvendo discentes e docentes. 


\section{CONCLUSÃO}

A possibilidade de colaborar com a compreensão das pesquisas e publicações sobre educação a distância no Brasil, compendiando o conhecimento já produzido, mostrando lacunas, evidenciado deficiências e identificando avanços e inovações, levou-se a optar por um mapeamento dos artigos científicos publicados na Revista Brasileira de Aprendizagem Aberta e a Distância (RBAAD), por meio da técnica bilbiométrica. Dessa forma, o objetivo proposto para a pesquisa foi atingido com profundidade e imparcialidade.

Em termos gerais, os artigos publicados na RBAAD, no que concerne aos aspectos metodológicos de coleta de dados (Observação, questionário e entrevista), natureza da pesquisa (qualitativa ou quantitativa) e classificação quanto aos objetivos (exploratória, descritiva e explicativa), deixaram a desejar porque a maioria absoluta dos artigos não revelaram estes aspectos.

No que se refere à utilização de normas brasileiras ou normas estrangerias para citações e referencias, predominou a utilização de outras normas em relação as normas da Associação Brasileira de Normas Técnicas (ABNT).

A quantidade de páginas por artigo mais representativa ficou em torno de 8 a 14 páginas. A maioria dos artigos, com $54 \%$ da amostra, teve apenas um autor por artigo.

Foram identificadas cerca de 11 (onze) áreas temáticas nos artigos da revista, sendo que $22 \%$ foi de Propostas Pedagógicas, 18\% envolvendo aspectos da Tecnologia da Informação e Comunicação (TIC) e experiências de EAD em outros países com 10\%.

Constatou-se que a RBAAD vem se firmando com artigos com conteúdos de qualidade, com novo padrão de layout depois da $10^{\mathrm{a}}$ edição, mas que demonstra deficiência, provavelmente do Conselho Editorial, por não solicitar revisões de seus artigos, inclusive com o acréscimo de itens referentes à metodologia da pesquisa, visto que apenas $13 \%$ dos artigos estão com resumos completos e que $94 \%$ dos artigos estão sem a especificação da natureza das pesquisas.

Por fim, sugere-se que novas pesquisas bibliométricas sejam realizadas no periódico para verificação da qualidade dos conteúdos, aplicação da lei de Lotka para os autores, determinação dos tipos de referências (livros, trabalhos monográficos e artigos) e autores mais citados.

\section{REFERÊNCIAS BIBLIOGRÁFICAS}

ABED - ASSOCIAÇÃO BRASILEIRA DE ENSINO A DISTÂNCIA. Revista Brasileira de Aprendizagem Aberta e a Distância (RBAAD). Disponível em: $<$ http://www.abed.org.br/revistacientifica/_brazilian/default.htm $>$. Acesso em: 10 jul 2013. 
AVELAR, Ewerton Alex; SANTOS, Thiago de Sousa; MESQUITA, Daniel Leite. Marketing: uma análise das pesquisas brasileiras desenvolvidas na primeira década do século XXI. XIV Semead - Seminário de Administração. São Paulo, USP, outubro de 2011.

CAPES - COORDENAÇÃO DE APERFEIÇOAMENTO DE PESSOAL. DE NÍVEL SUPERIOR. Classificação Qualis. Disponível em: < http://qualis.capes.gov.br /webqualis/principal.seam>. Acesso em: 19 set. 2013.

CARPES, Aletéia de Moura. Uma Análise da Produção Científica Brasileira sobre Educação a Distância. Anais do VIII Simpósio de Excelência em Gestão e Tecnologia. Resende, 21 de outubro de 2011.

CASTELLS, Manuel. A Sociedade em Rede. 6. ed. São Paulo: Paz e Terra, 1999.

CERVO, Amado Luiz; BERVIAN, Pedro Alcino. Metodologia Científica. 5 ed. São Paulo: Prentice Hall, 2002.

DIEHL, Astor Antônio; TATIM, Denise Carvalho. Pesquisa em Ciências Sociais. São Paulo: Prentice Hall, 2004.

GIDDENS, Anthony. As Consequências da Modernidade. São Paulo: Editora Unesp, 1991.

GIDDENS, Anthony. Modernidade e Identidade. Rio de Janeiro; Editora Jorge Zahar, 2002.

GIL, Antônio Carlos. Métodos e técnicas de pesquisa social. 4 ed. São Paulo: Atlas, 1996.

GUEDES, Vânia L; BORSCHIVER, Suzana. Bibliometria: Uma ferramenta estatística para a gestão da informação e do conhecimento, em sistemas de informações, de comunicação e de avaliação científica e tecnológica. Disponível em: $<$ http:// https://www.google.com.br/\#q=bibliometria+uma+ferramenta+ estat $\% \mathrm{C} 3 \% \mathrm{ADstica}+$ para $+\mathrm{a}+$ gest $\% \mathrm{C} 3 \% \mathrm{~A} 3 \mathrm{o}+\mathrm{da}+$ informa $\% \mathrm{C} 3 \% \mathrm{~A} 7 \% \mathrm{C} 3 \% \mathrm{~A} 3 \mathrm{o}>$. Acesso em 13 set. 2013.

MOORE, Michael; KEARSLEY, Greg. Educação a Distância. São Paulo> Thomson Learning, 2007.

MOURA, Geovanne Dias de; DALLABONA, Lara Fabiana; LAVARDA, Carlos Eduardo Facin. Perfil dos estudos sobre o tema Orçamento publicados em congressos brasileiros, de 2005 a 2009. Revista Contabilidade Vista \& Revista, Belo Horizonte, UFMG, v. 23, n. 1, p. 97-125, jan./mar. 2012.

RIBEIRO, Henrique Cesar Melo et al. Visão Baseada em Recursos: Uma análise Bibliométrica dos últimos 11 anos. Revista Ciências da Administração. v.14, n.34, p. 39-59, dez. 2012. 
SERRA NEGRA, Carlos Alberto. Normas Internacionais de Referências

Bibliográficas. Porto Alegre: Cidadela, 2011.

SPANHOL, Fernando José et al. O estado da Arte da Educação a Distância: Uma Meta-Análise da Contribuição da UFSC. Disponível em: http://www.abed.org.br /congresso2010/cd/252010180446.pdf>. Acesso em: 12 ago. 2013. 